number of cases within a limited time in a small area that the influence of weather can be tested. This is what $I$ have done. So far as I know, it is the first attempt of the kind in Europe.

In New York, Seibert, by means of a collective investigation, has carried out a similar inquiry. He took the year March, 1884, to March, 1885, and collected over 700 cases. He found February and March to be the most pneumonic months in the year, and concluded that the three chief climatic factors were $(a)$ a low and sinking temperature, $(b)$ a high and rising humidity, $(c)$ a strong wind, and brings pneumonia into line with bronchitis. None of these conclusions are borne out by my tables; they certainly do not harmonise with European statistics and opinions, and, judging from Osler's sentence above quoted, they do not agree much better with those of America. The tables are not given in detail, so that it is impossible to compare them with my own.

Of course I do not suppose that in atmospheric conditions we shall find all the factors that cause pneumonia. Even leaving aside the pneumococcus, the weather does not explain epidemics, and it probably does not act similarly in all climates. Still, it is undoubtedly the general opinion that it has a considerable influence, and it will therefore be useful to have shown with some accuracy the conditions which last year in London varied with the prevalence of the disease.

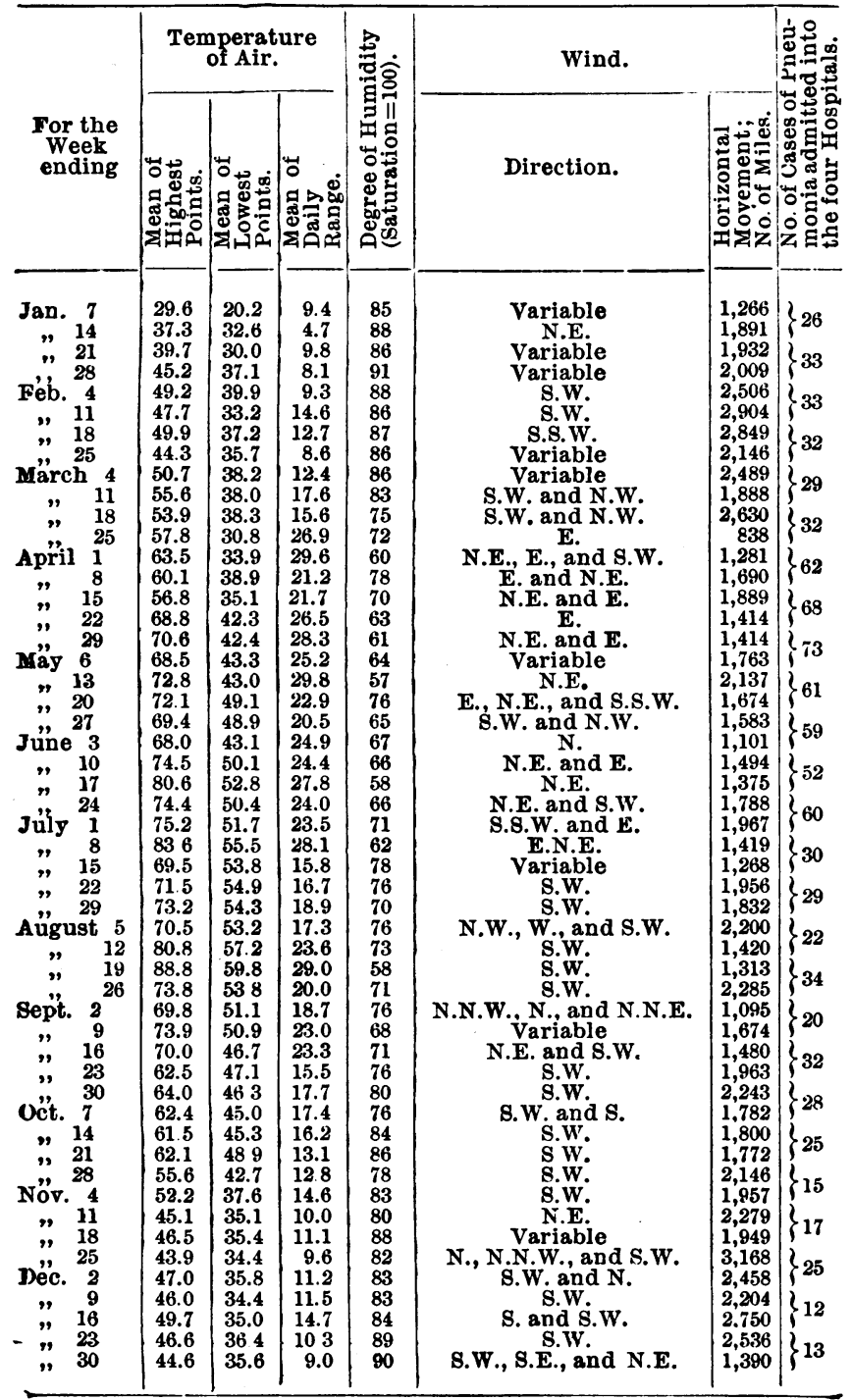

\section{A CASE OF PERFORATION OF A CHRONIC ULCER OF THE DUODENUM SUCCESSFULLY TREATED BY EXCISION.}

DEATH TWO MONTHS LATER FROM ACUTE INTESTINAL OBSTRUCTION BY A BAND.

By HENRY PERCY DEAN, M.S., B.Sc.LoNd., F.R.C.S., Assistant Surgeon to the London Hospital; Surgeon to North-Eastern Hospital for Children.

E. F., a married woman, aged 27 years, was admitted into one of the surgical wards of the London Hospital on the evening of February 17th, 1894. She was seen by me about 1.30 A.M. on the following morning, that is, a few hours after her admission, and I found her in the following condition :

Intense pain was complained of over the whole of the abdomen, perhaps slightly more marked in the epigastric regions. The patient felt very ill,, and her expression was exceedingly anxious. Vomiting occurred every few minutes, and much exhausted the patient. The pulse was 120 , feeble and regular. The respirations were rapid and irregular both in force and rhythm. The tongue was slightly furred, and very dry. There was uniform tenderness over the whole abdomen, the distension was moderate in degree, and the resonance of the percussion note was markedly increased. The temperature was $1006^{\circ}$.

On questioning the patient it was found that for about fortnight she had suffered from pain in the chest and pit of stomach, and that her doctor had been treating her for indigestion. The patient had been sick several times during the week previous to her admission, and her bowels had not been opened for seven or eight days. About twenty-four hours before admission to the hospital the patient suddenly became much worse, feeling a severe pain in the pit of the stomach, and a sensation of intense weakness. This was soon followed by vomit ng, repeated at frequent intervals.

It secmed to me pretiy evident that the patient was suffering from acute general peritonitis. The possibility of perforation of the stomach or duodenum was considered, but on. the whole I was rather inclined to think that it was a case of mechanical obstruction with general peritonitis. It was decided to operate at once. The patient was taken to the operating theatre, anæsthetised with chloroform, and an incision about 3 inches long was made immediately below the umbilicus. On opening the peritoneal cavity a quantity of purulent fluid escaped, and coils of intestine, somewhat distended, intensely congested, and covered in places with flakes of lymph, protruded through the wound. No evidence of any mechanical obstruction could be obtained, so $I$ increased the incision upwards to the ensiform cartilage, and in a few minutes I found some flakes of lymph in the region. of the gall bladder. In the centre of one of these flakes I noticed come gas bubbles, forming a kind of froth. On inserting a probe into the froth $I$ found that it passed into a cavity, which on further examination was found to be the duodenum, the perforation being situated on the anterior aspect about $\frac{3}{4}$ inch beyond the pylorus. Around the perforation a distinct induration could be felt. By means of scissors this indurated area was excised. The portion removed was elliptical in shape, measuring $1 \frac{1}{4}$ inch in its long axis, which was parallel with the transverse axis of the gut. The portion excised was found to include the ulcer and a margin of healthy mucous membrane. In the centre of the ulcer was a perforation about $2 \mathrm{~mm}$. in diameter. The floor of the ulcer was white, and the edge of the ulcer was bounded by apparently healthy mucous membrane. The elliptical opening thus made into the duodenum was sewn up by silk sutures according to Lembert's method. The peritoneal cavity was washed out with warm weak boracic lotion, well sponged, and the wound sewn up by stitches passing throughm the whole thickness of the abdominal wound. The operation lasted fifty minutes.

After the operation the patient was allowed nothing by the mouth. She was fed solely per rectum by nutrient enemata and suppositories for seventeen days. To allay thirst, 2 ounces of warm water were occasionally injected into the 
rectum. The patient rapidly improved, was given liquid food by the mouth on the eighteenth day, and solid food on the twenty-eighth day. The abdominal wound healed by first intention, and on March 19th, that is, thirty days after the operation, the patient was walking about the ward, going into the garden, and eating ordinary food.

It was decided to send the patient into the country, and so she was kept in the hospital until a vacancy in a convalescent home occurred. Three days before the date on which it was arranged that she should leave the hospital, that is, on the evening of April 13th, the patient complained of some pain in the abdomen, and was sick. The pain continued on Saturday morning, and she was sick again. I saw the patient in the afternoon of Saturday, and found her complaining of a colicky pain in the abdomen. Her general condition was good. I ordered an enema to be given, and all food by the mouth to be stopped. Having arranged to leave London on that day for a short holiday, I asked the house. surgeon to call in one of my colleagues, if the patient's condition did not rapidly improve. No result followed the enema, so the house-surgeon ordered doses of mag. sulph. ( $3 j$ ) to be given every hour. Four of these doses were given, and on Sunday morning the patient was so much worse that Mr. Eve was asked to see the patient. Mr. Eve considered that she was suffering from intestinal obstruction, probably caused by a band, and proceeded- to perform abdominal section. Ether was administered, and an incision was made through that part of the scar of the former incision that was below the umbilicus. The great omentum was found adherent to the wound, and at the lower part of the scar a band could be traced to a coil of intestine, and this band had caused a kink in the gut sufficient to produce complete obstruction. The band was attached to the gut about 6 inches above its en trance into the cæcum. The coils of intestine on the proximal side of the band were greatly distended and very congested. On the distal side of the band the intestine was collapsed. The band was removed, and immediately afterwards the collapsed coils became distended, showing that the obstruction had been relieved. The peritoneal cavity, which contained some blood-stained fluid, was washed out with warm boracic lotion, and the wound sewn up by silkworm-gut stitches. The operation lasted 45 minutes.

The patient recovered to a slight extent from the shock of the operation, but never thoroughly rallied. She gradually became weaker, and died about thirty-six hours after the operation.

Post-mortem Examination.-On opening the abdomen a considerable amount of lymph was found upon the intestines, and the peritoneal cavity contained a quantity of turbid fluid. About 3 inches above the attachment of the band to the small intestine was a perforation from which the intestinal contents escaped. A little higher up was another perforation. On opening the intestine it was found that each perforation was in the centre of a small ulcer. The ulcers were evidently of very acute and recent formation, as the whole thickness of the intestinal wall at these spots was necrosed. There were several other necrotic patches within 18 inches of the obstructed point. For about half an inch from the pylorus the duodenum was thinner than normal, and the peritoneum over it was puckered. The duodenum was quite healthy, and presented no traces of past or recent ulceration.

REMARKs. - This case is of interest from two distinct points of view. First, the successful treatment of the duodenal ulcer; secondly, the unfortunate sequela of strangulation by a band. More especially is the case of interest from the first point of view, because, so far as I have been able to ascertain, no successful result of excision of a perforated duodenal ulcer has yet been recorded. Not long ago Mr. Lockwood read a paper before this Society on two cases of perforation of duodenal ulcers, and in each of which he failed to find the perforation. In his cases the perforation was on the posterior wall of the duodenum, and almost impossible to expose. In my case, on the other hand, the perforation was situated in the most convenient spot that could be desired-namely, on its anterior aspect, and the difficulties to be overcome in excising the ulcer and suturing the duodenum were but olight.

As regards the unfortunate result, it is impossible, so far as
I can see, to prevent the formation of a band after abdominal section for acute peritonitis, especially acute purulent peritonitis. In these purulent cases, at any rate, I do not think that stitching up the peritoneum by itself, before suture of the abdominal wall, could lessen in any way the tendency to the formation of adhesions between the inflamed, lymphcovered intestines and the abdominal wall.

One is rather inclined to conclude with this statementthat if after abdominal section an attack of acute abdominal pain and intestinal obstruction supervene, an exploratory operation should be performed as soon as possible, even if the general condition of the patient would, under ordinary circumstances, scarcely be considered grave enough for immediate surgical interference.

I wish to express my thanks and indebtedness to my colleague, Mr. Eve, for his kindness in taking charge of the patient during my absence.

\section{HALLUCINATIONS IN THE SANE, ASSOCIATED WITH LOCAL ORGANIC DISEASE OF THE SENSORY ORGANS, ETC.}

Bx W. S. COLMAN, M.D., M.R.C.P., Assistant Physician to the Hospital for Sick Children; Registrar to the National Hospital for the Paralysed and Epileptic.

THE following paper deals with cases which have come under observation during the last six years at the National Hospital for the Paralysed and Epileptic, and $I$ am indebted to the physicians under whose care they were for permission to make use of the notes.

Inasmuch as there was a definite peripheral cause for the hallucinations, the more restricted term "illusion" might have been more accurate, but as this would lead to confusion in the cases where local ear disease led to hallucinations of vision as well as hearing, the more general term "hallucination" has been employed throughout, it being now pretty generally recognised that there is no essential difference in the nature of illusions and hallucinations.

Hallucinations due to drugs (alcohol, opium, haschish), to toxic substances circulating in the blood (as in fevers, etc.), those associated with thoracic or abdominal visceral disease, and those occurring as an aura in rare cases of idiopathic epilepsy are purposely omitted. Attention is confined to those cases in which there was demonstrable local disease of the eye, ear, etc., or of sensory regions of the brain.

It will be noticed that the hallucinations were mostly temporary, associated with some general bodily disturbance, and passed off with the return of the general health to normal. So, too, the whole brain seems to have been disturbed, and we find hallucinations excited of other senses than the one whose organs are affected.

Hallucinations due to Local Diseases of the Eye.

These are far from infrequent. They are usually extremely definite, so that the patient can give an extremely vivid and detailed account of them. Unless he is much out of health, he rarely believes in their reality, in marked contrast to the condition in toxic cases (alcohol, etc.).

They occur frequently from opacities in the media, and occasionally in glaucoma seem to represent the flashes of light which are so often experienced in that disease. They are common in cases of optic atrophy, but I have not at present met with them associated with optic neuritis.

CASE I. Old Choroiditis, and Floating Bodies in Field of Vision: Fresh InCASE I. Old Choroiditis, and Floating Bodies in Field of Vision: Fresh In
flammation of Eye: Temporary Hallucinations of Vision.-Miss C., aged 38 flammation of Eye: Temporary Hallucinations of Lision.-Miss C., aged 3 (under the care of Dr. Ferrier), had had choroidits and Hoating or narin the vitreous for some years. She had not been taking alcohol or nar cotics. Coincidently with some acute inflammation of the eyes, which depressed her very much, she developed hallucinations or vision. She complained that numerous beetles like ordinary cockroaches with red eyes kept swarming over the bed and on the ceilyg. Two days later she complained in addition that " a woman in red, all on fre, kept coming to her bedside, and annoying her (one of the nurses in ordinary uniform). For a day or two she believed in the reality of these appearances, but as her general condition improved, she ceased to mind them. When the disappeared, the field again became full of ordinarv muscæ volitantes. CASE II. Syphilitic Retinitis: Numerous Floating Bodies in Field:Temporary Hallucinations of Vision.-W. E., aged 50. The retinitis noted above was of long standing, and had been quiescent for some years, but had recently become troublesome, and for some months he had had numerous 